\title{
Ortodontia preventiva e interceptativa na rede de atenção básica do SUS: perspectiva dos cirurgiões-dentistas da Prefeitura Municipal de Florianópolis, Brasil
}

\author{
Preventive and interceptive orthodontics in the primary health \\ care network of the Brazilian Unified Health System: \\ the viewpoint of the dental surgeons of Florianopolis City Hall
}

Samuel Carlos Guzzo ${ }^{1}$

Mirelle Finkler ${ }^{1}$

Calvino Reibnitz Júnior ${ }^{1}$

Marynes Terezinha Reibnitz ${ }^{2}$

${ }^{1}$ Departamento de Odontologia, Universidade Federal de Santa Catarina. Campus Universitário, Trindade. 88.040-970 Florianópolis SC Brasil. samuel_gusso_1@hotmail.com ${ }^{2}$ Secretaria Municipal de Saúde da Prefeitura de Florianópolis.

\begin{abstract}
The scope of this exploratory study was to understand the viewpoint of the dental surgeons of the Primary Health Care (PHC) network in Florianópolis regarding the need, feasibility and interest in broadening preventive orthodontic services and in the implementation of interceptive orthodontics. A structured questionnaire was used to establish the viewpoint of all the primary care dental surgeons who perform clinical care in the network. The results indicate that: the majority are in favor provided that there is an appropriate structure to attend the needs of the population; the professionals consider themselves unqualified to perform the requisite procedures, so training would be necessary; there would be no need to deploy other orthodontic procedures in primary care than those analyzed; and the main difficulties that might be encountered were related to excess demand and the lack of human resources. Despite these obstacles, the majority of the professionals consider it positive and feasible to introduce this service, which could increase the comprehensiveness of health care and bolster primary care, thereby improving the health of the population.
\end{abstract}

Key words Public health, Primary Health Care, Unified Health System, Malocclusion; Orthodontics
Resumo O objetivo deste estudo exploratório foi conhecer a perspectiva dos cirurgiões-dentistas da rede de atenção básica à saúde de Florianópolis acerca da necessidade, viabilidade e interesse quanto à ampliação dos serviços de ortodontia preventiva e à implementação dos de ortodontia interceptativa nas Unidades Básicas de Saúde do município. Empregou-se um questionário estruturado para coletar as opiniões de todos os cirurgiõesdentistas da atenção básica que realizam atendimento clínico na rede. Os resultados demonstraram que: a maioria deles é favorável desde que haja uma estruturação adequada para atender à necessidade da população; que os profissionais se consideram despreparados para realizar os procedimentos necessários, de modo que haveria a necessidade de capacitações; que não haveria a necessidade de se implantar outros procedimentos de ortodontia na atenção básica além dos analisados; e que as principais dificuldades que seriam enfrentadas estariam relacionadas ao excesso de demanda e à falta de recursos humanos. Apesar destes obstáculos, a maioria dos profissionais encara como positiva e viável a ampliação/introdução desse serviço, o que favoreceria a integralidade da atenção à saúde e fortaleceria a atenção básica, melhorando assim a saúde da população.

Palavras-chave Saúde coletiva, Atenção Básica de Saúde, SUS, Oclusopatias, Ortodontia 


\section{Introdução}

Devido às ações de políticas públicas em saúde bucal, tais como a fluoretação das águas de abastecimento e dos dentifrícios ${ }^{1}$, bem como o crescente acesso da população ao atendimento odontológico, observam-se notáveis transformações nos padrões epidemiológicos das enfermidades e agravos à saúde bucal com um grande declínio da doença cárie, fazendo com que outras morbidades e condições comecem a ganhar maior importância para a saúde pública.

As oclusopatias atualmente ocupam a terceira posição em uma escala de prioridades dos problemas bucais ${ }^{2}$. Constituem-se de anomalias do crescimento e desenvolvimento dos músculos e ossos maxilares no período da infância e adolescência, que podem produzir alterações tanto do ponto de vista estético, quanto do funcional da oclusão, mastigação, fonação, respiração e deglutição, além de contribuir na postura corporal ${ }^{3}$.

A oclusão dentária é importante para a manutenção de todo o equilíbrio biológico do indivíduo ${ }^{4}$. Interfere na qualidade de vida e pode influenciar negativamente no que se refere aos fatores psicossociais, uma vez que um sorriso desarmonioso pode ser motivo de constrangimento nos relacionamentos afetivo-familiares e no ambiente social ${ }^{5}$.

O diagnóstico precoce de condições que influenciam o desenvolvimento normal da oclusão dentária pode contribuir para diminuir significativamente a incidência de maloclusões ${ }^{6}$. Utilizando-se procedimentos simples de Ortodontia preventiva e interceptativa, como aqueles que objetivam a manutenção de espaço ou a sua recuperação, o controle de hábitos bucais deletérios, e o tratamento da mordida cruzada e da mordida aberta, pode-se prevenir ou amenizar a severidade das oclusopatias ${ }^{7}$. Do ponto de vista clínico, é necessário diagnosticar e intervir precocemente e de forma adequada em benefício da evolução normal da dentição e do crescimento crânio-facial ${ }^{8}$, quando as oclusopatias instaladas não sofrerão autocorreção com a maturação da oclusão, ou seja, quando não se tratar de uma condição clínica transitória ${ }^{7}$.

Tendo em vista os possíveis prejuízos das oclusopatias, torna-se preocupante a falta de acesso ao seu tratamento à maior parte da população ${ }^{9}$. $\mathrm{O}$ tratamento de mordidas cruzadas, o controle de hábitos deletérios e pequenos movimentos dentários na fase de dentição mista, em Unida- des Básicas de Saúde (UBS), praticamente inexiste. A pesquisa realizada por Castro $^{10} \mathrm{com} 211$ cirurgiões-dentistas (CD) de oito municípios catarinenses mostrou que entre $95,7 \%$ e $97,1 \%$ dos $\mathrm{CD}$ nunca realizaram tais procedimentos. Manutenção e recuperação de espaço também nunca são realizados por respectivamente $61,6 \% \mathrm{e}$ $79,4 \%$ deles.

Desta forma, o panorama da atenção às oclusopatias no Brasil caracteriza-se por elevada prevalência e insuficiente capacidade de cobertura. Mesmo nas regiões mais desenvolvidas, como a Sul, onde o número de especialistas em ortodontia por habitantes é de um para 8,56 mil, há uma insuficiência na cobertura das necessidades epidemiológicas, pois a capacidade da rede privada atender a demanda de tratamentos fica restrita à pequena parcela da população que possui condições financeiras para acessá-lo ${ }^{10}$.

Disponibilizando-se serviços ortodônticos preventivos e interceptativos já na atenção básica $(\mathrm{AB})$, facilitar-se-ia o acesso da população ao tratamento, possibilitando a eliminação de alguns fatores etiológicos e prevenindo a progressão das desarmonias dentárias, esqueléticas e funcionais, minimizando ou até mesmo eliminando a necessidade de tratamentos mais complexos posteriormente. Para tanto, Hebling et al. ${ }^{7}$ estruturaram um protocolo de atenção às oclusopatias na $\mathrm{AB}$, indicando quais condições deveriam ser consideradas prioritárias a tratar, quais tratamentos estariam indicados para cada condição, bem como a documentação ortodôntica necessária. Trata-se de um protocolo pioneiro, mas que demonstra a viabilidade de se realizar ações ortodônticas preventivas e interceptadoras nas UBS nas fases de dentadura decídua e mista a partir de uma adequada organização do serviço.

Diante do exposto, este estudo objetivou conhecer a perspectiva dos $\mathrm{CD}$ que trabalham na rede de $\mathrm{AB}$ à saúde de Florianópolis sobre a ampliação dos serviços de ortodontia preventiva (uma vez que o controle de hábitos bucais deletérios é assim classificado e já vem sendo realizado na $A B)$ e sobre a implementação dos serviços de ortodontia interceptativa. Mais especificamente, buscou-se levantar as necessidades sentidas por tratamentos ortodônticos preventivos e interceptativos; identificar os procedimentos ortodônticos mais relevantes para a $\mathrm{AB}$; conhecer as potencialidades e as dificuldades detectadas pelos $\mathrm{CD}$ em viabilizar o serviço na rede; além de conhecer seu interesse em realizar este tipo de tratamento. 


\section{Metodologia}

Foi realizado um estudo exploratório transversal (aprovado pelo CEPSH/UFSC), para o qual foram solicitados a participar como sujeitos de pesquisa todos os $71 \mathrm{CD}$ que atuam clinicamente na rede de $\mathrm{AB}$ à saúde do município de Florianópolis. Um total de 64 profissionais $(90,14 \%)$ aceitou participar após receberem explicações e terem lido e assinado um Termo de Consentimento Livre e Esclarecido.

Os dados foram coletados através de questionário semiestruturado autoaplicado, composto por questões fechadas, abertas e mistas. O instrumento continha ainda, um breve enunciado para contextualizar os sujeitos quanto aos resultados do último levantamento epidemiológico de cobertura nacional sobre as condições de saúde bucal, bem como sobre a publicação da Portaria $n^{\circ} 718 / S^{-11} S^{11}$ que ampliou o rol de procedimentos ortodônticos na atenção secundária e terciária, e que incluiu a possibilidade de alguns procedimentos ortodônticos serem realizados também na atenção básica.

Após o retorno dos questionários, as respostas às questões fechadas foram compiladas em planilhas eletrônicas e analisadas através de estatística descritiva e do método de correlação de Spearman, considerando-se estatisticamente significativos os resultados $\operatorname{com} p<0,05$. Já as respostas às questões abertas foram digitalizadas em documentos eletrônicos e categorizadas de acordo com suas ideias centrais.

\section{Resultados e discussão}

Metade dos sujeitos de pesquisa (32) possui até 15 anos de atuação profissional. Aproximadamente um terço $(34,4 \%)$ dos $\mathrm{CD}$ (22) trabalham a menos de cinco anos na $\mathrm{AB}$, outro terço aproximado (34,5\%) trabalha de 5 a 20 anos (22), e os demais (31,3\%), a mais de 20 anos (20). Quanto à formação dos entrevistados, a maioria $(76,6$ $\%)$ possui algum curso de pós-graduação (49). Destes, a maior parte $(79,6 \%)$ possui especialidade na área da saúde coletiva (39), sendo 59,2\% somente na área de saúde coletiva (29) e 20,4\% na área da saúde coletiva e em alguma área clínica (10).

\section{Necessidade percebida de se realizar tratamentos ortodônticos preventivos e interceptativos na rede de $\mathrm{AB}$ à saúde}

Conforme se pode observar na Tabela 1 , a maioria dos CD classificou como "média" a necessidade de se realizar os tratamentos elencados no questionário, quais sejam de manutenção de espaço $(57,8 \%)$, recuperação de espaço $(48,4 \%)$, controle de hábitos bucais deletérios $(39,1 \%)$, tratamento de mordida cruzada (51,6 \%) e de mordida aberta $(54,7 \%)$. Outro dado observado foi uma tendência a considerar os tratamentos no sentido de "média" necessidade para "pouca" necessidade, com exceção do controle de hábitos bucais deletérios, para o qual houve indicação no sentido de "média" para "muita" necessidade.

Estes resultados condizem com os estudos epidemiológicos que mostram uma considerável prevalência de oclusopatias em crianças de todo o país. Segundo o último levantamento epidemi-

Tabela 1. Perspectiva dos $\mathrm{CD}$ da rede de $\mathrm{AB}$ quanto à necessidade de se realizar tratamentos ortodônticos preventivos e interceptativos nas UBS.

\begin{tabular}{|c|c|c|c|c|c|c|c|c|c|c|}
\hline & \multicolumn{2}{|c|}{ Man. espaço } & \multicolumn{2}{|c|}{ Rec. espaço } & \multicolumn{2}{|c|}{ C.H.B.D. } & \multicolumn{2}{|c|}{ Mord. cruzada } & \multicolumn{2}{|c|}{ Mord. aberta } \\
\hline & $F$ & $\mathbf{P}$ & F & $\mathbf{P}$ & $\mathbf{F}$ & $\mathbf{P}$ & $\mathbf{F}$ & $\mathbf{P}$ & $F$ & $\mathbf{P}$ \\
\hline Desconheço & 3 & 4,7 & 4 & 6,3 & 2 & 3,1 & 2 & 3,1 & 2 & 3,1 \\
\hline Nenhuma & - & - & 1 & 1,6 & 1 & 1,6 & - & - & 1 & 1,6 \\
\hline Pouca & 12 & 18,8 & 16 & 25,0 & 17 & 26,6 & 22 & 34,4 & 17 & 26,6 \\
\hline Média & 37 & 57,8 & 31 & 48,4 & 25 & 39,1 & 33 & 51,6 & 35 & 54,7 \\
\hline Muita & 11 & 17,2 & 12 & 18,8 & 18 & 28,1 & 7 & 10,9 & 9 & 14,1 \\
\hline Total & 63 & 98,4 & 64 & 100 & 63 & 98,4 & 64 & 100,0 & 64 & 100,0 \\
\hline Perdas & 1 & 1,6 & - & - & 1 & 1,6 & - & - & - & - \\
\hline Total & 64 & 100,0 & 64 & 100,0 & 64 & 100,0 & 64 & 100,0 & 64 & 100,0 \\
\hline
\end{tabular}

$\mathrm{F}=$ frequência absoluta, $\mathrm{P}=$ percentual. 
ológico nacional ${ }^{12}$ que empregou o índice de Roster e Hamiltton, aos 5 anos de idade, $66,7 \%$ das crianças apresentaram pelo menos uma condição de oclusopatia. Aos 12 anos, 38\% apresentaram problemas de oclusão, segundo o índice DAI, sendo em $20 \%$ na forma mais branda, em $11 \%$ na forma mais severa, e em $7 \%$, muito severas, requerendo tratamento imediato.

Em São Paulo, levantamento realizado em 1996 estimou a prevalência dos problemas oclusais, constatando $48,97 \%$ aos 5 anos e $71,31 \%$ aos 12 anos. Considerando-se as anomalias moderadas ou severas, conforme critérios preconizados pela Organização Mundial da Saúde em 1987, o percentual foi de respectivamente $26,12 \%$ na dentição decídua e $39,79 \%$ na mista ${ }^{13}$.

Na cidade de Salvador (BA), em 2004, um estudo com escolares de 12 a 15 anos constatou, a partir da aplicação do índice DAI, que $45,76 \%$ apresentaram necessidade de tratamento ortodôntico, sendo $26,29 \%$ de tratamento "eletivo", 8,29\% de "desejável" e 11,18\% de "mandatório"14. Já em Belo Horizonte (MG), em 2003, a prevalência de maloclusão foi de $62,2 \%$ aos 10 e 12 anos e de $61,3 \%$ aos 13 e 14 anos. A necessidade normativa de tratamento ortodôntico a partir do índice DAI foi de $52,3 \%{ }^{15}$.

Constata-se, portanto, a importância da assistência aos indivíduos com oclusopatias e sua necessária inserção na $\mathrm{AB}$ à saúde ${ }^{9,15}$, mas não sem se observar a opinião dos sujeitos a respeito (critérios subjetivos autopercebidos), uma vez que se sabe que os critérios normativos (tecnicamente definidos) para tratamento ortodôntico superestimaram a prevalência dos problemas oclusais quando comparados à percepção das pessoas. É preciso se considerar que na sociedade ocidental, a demanda por tratamento ortodôntico é um fenômeno cultural (parcialmente mediado pela capacidade de pagamento), de modo que a decisão sobre intervir ou não deve ponderar também o significado cultural e social do aspecto dento-facial para cada indivíduo ${ }^{16}$.

Ainda com relação à necessidade percebida pelos $C D$ dos tratamentos ortodônticos na $A B$, vale atentar para a relação inversamente proporcional entre o tempo de atuação profissional e na $\mathrm{AB}$, e a necessidade percebida para todos os tratamentos citados (Tabela 2), ainda que apenas para manutenção de espaço tal correlação $(\rho=$ $-0,358)$ tenha apresentado significância estatística. Em outras palavras, quanto maior o tempo de atuação profissional e na $\mathrm{AB}$, menor a necessidade sentida pelos CD de tratamentos ortodônticos nas UBS. Algumas possíveis explicações para este fato poderiam ser um maior distanciamento destes profissionais da formação acadêmica em termos de tempo, ou mesmo a ausência do enfoque ortodôntico preventivo na formação, ou ainda, uma maior percepção de outras prioridades em termos de saúde bucal à medida que estão a mais tempo envolvidos com o SUS. Mas seria necessária uma abordagem metodológica diferente da empregada para se compreender melhor tais fatos.

Já os cruzamentos entre as necessidades percebidas de realização dos diferentes tipos de tratamentos apresentaram correlações positivas e significância estatística (Tabela 2), exceto o cruzamento entre necessidade de manutenção de espaço e de tratamento para mordida cruzada, que, apesar de não possuir valor de $p$ significativo também se relacionam positivamente $(p=0,31)$. Em outras palavras, quando um CD considera importante a realização de um determinado tratamento, os demais também são por ele considerados importantes.

\section{Aptidão para realizar os tratamentos ortodônticos preventivos e interceptativos}

A maioria dos CD respondeu sentir-se "pouco apta" para realizar os tratamentos elencados no questionário (Tabela 3 ), sendo os percentuais de $51,5 \%$ para manutenção de espaço, $59,4 \%$ para recuperação de espaço, $54,7 \%$ para controle de hábitos bucais deletérios, 53,1\% para mordida cruzada e $59,4 \%$ para mordida aberta.

Tais dados corroboram os resultados obtidos por Castro ${ }^{10}$, onde especificamente para os procedimentos que envolvem a instalação de aparelhos ortodônticos, a maioria dos CD se considera pouco capacitada, muito pouco capacitada ou não capacitada, sendo os percentuais de 69,38\% para correção da mordida cruzada anterior, seguido da instalação de aparelho para correção da mordida cruzada posterior $(68,90 \%)$, para recuperação de espaço $(67,31 \%)$, para correção de hábitos bucais $(66,99 \%)$ e para manutenção de espaço (57,89\%). Desta forma, evidencia-se que os profissionais se sentem despreparados para tais procedimentos.

Neste estudo, chama também a atenção um percentual significativo de $\mathrm{CD}$ que se considera "nada apto" para realizar manutenção de espaço, recuperação de espaço, tratamento de mordida cruzada e de mordida aberta $(21,9 \%, 31,3 \%$, $28,1 \%$ e $29,7 \%$, respectivamente). Já em relação ao controle de hábitos bucais deletérios, houve um posicionamento diverso: $21,9 \%$ dos CD se 
Tabela 2. Correlação de Spearman e teste de significância estatística no cruzamento entre as variáveis.

\begin{tabular}{|c|c|c|c|c|c|c|}
\hline & $\begin{array}{c}\text { Tempo } \\
\text { atuação } \\
\text { profissional }\end{array}$ & $\begin{array}{c}\text { Tempo } \\
\text { AB }\end{array}$ & $\begin{array}{c}\text { Necess. } \\
\text { man. } \\
\text { espaço }\end{array}$ & $\begin{array}{l}\text { Aptidão } \\
\text { man. } \\
\text { espaço }\end{array}$ & $\begin{array}{c}\text { Necess. } \\
\text { rec. } \\
\text { espaço }\end{array}$ & $\begin{array}{c}\text { Aptidão } \\
\text { rec. } \\
\text { espaço }\end{array}$ \\
\hline \multicolumn{7}{|c|}{ Tempo atuação profissional } \\
\hline correlação $\rho$ & 1,000 &, 884 &,- 358 &,- 366 &,- 188 &,- 250 \\
\hline significância p & . &, 000 &, 004 & ,003 &, 136 & ,046 \\
\hline \multicolumn{7}{|l|}{ Tempo AB } \\
\hline correlação $\rho$ &, 884 & 1,000 &,- 285 &,- 382 &,- 037 &,- 212 \\
\hline significância $\mathbf{p}$ &, 000 & . & ,024 & ,002 &, 772 & 092 \\
\hline \multicolumn{7}{|c|}{ Necessidade manutenção espaço } \\
\hline correlação $\rho$ &,- 358 &,- 285 & 1,000 & ,092 &, $543^{* *}$ & 088 \\
\hline significância $\mathbf{p}$ &, 004 &, 024 & . & ,477 &, 000 & ,493 \\
\hline \multicolumn{7}{|c|}{ Aptidão manutenção espaço } \\
\hline correlação $\rho$ &,- 366 &,- 382 & 092 & 1,000 &, 010 & 646 \\
\hline significância $\mathbf{p}$ & ,003 & ,002 & ,477 & . & 940 &, 000 \\
\hline \multicolumn{7}{|c|}{ Necessidade rec. espaço } \\
\hline correlação $\rho$ &,- 188 &,- 037 &, 543 &, 010 & 1,000 & 078 \\
\hline significância $\mathbf{p}$ &, 136 &, 772 &, 000 & ,940 & . &, 539 \\
\hline \multicolumn{7}{|l|}{ Aptidão rec. espaço } \\
\hline correlação $\rho$ &,- 250 &,- 212 & 088 & ,646 &, 078 & 1,000 \\
\hline significância $\mathbf{p}$ &, 046 & ,092 & ,493 &, 000 &, 539 & . \\
\hline \multicolumn{7}{|c|}{ Necessidade controle hábitos deletérios } \\
\hline correlação $\rho$ &,- 149 &,- 065 & ,363 & 099 &, $458^{* *}$ &, 189 \\
\hline significância $\mathbf{p}$ & ,243 & 615 & 004 & ,444 &, 000 & 138 \\
\hline \multicolumn{7}{|c|}{ Aptidão controle hábitos deletérios } \\
\hline correlação $\rho$ &,- 221 &,- 128 &, 147 & ,591 & 182 & ,540 \\
\hline significância $\mathbf{p}$ &, 082 &, 316 & 254 &, 000 & 154 &, 000 \\
\hline \multicolumn{7}{|c|}{ Necessidade trat. M.C. } \\
\hline correlação $\rho$ &, 108 &, 129 &, 031 &,- 054 &, $358^{* *}$ &, 003 \\
\hline significância $\mathbf{p}$ & ,398 & 310 &, 811 &, 675 &, 004 & ,981 \\
\hline \multicolumn{7}{|l|}{ Aptidão trat. M.C. } \\
\hline correlação $\rho$ &,- 125 &,- 139 &,- 033 &, 552 &, 047 & ,758 \\
\hline significância $\mathbf{p}$ &, 324 & 275 & ,795 &, 000 &, 709 &, 000 \\
\hline \multicolumn{7}{|c|}{ Necessidade trat. M.A. } \\
\hline correlação $\rho$ &,- 063 &,- 051 & ,332 & 023 &, $362^{* *}$ & 099 \\
\hline significância $\mathbf{p}$ &, 620 & ,692 & 008 & 857 & ,003 & 434 \\
\hline \multicolumn{7}{|l|}{ Aptidão trat. M.A. } \\
\hline correlação $\rho$ &,- 219 &,- 199 &, 115 & ,662 & ,098 & ,745 \\
\hline significância $\mathbf{p}$ &, 082 &, 115 &, 368 &, 000 & 442 &, 000 \\
\hline
\end{tabular}

consideram "muito aptos" e 10,9\%, "totalmente aptos” (Tabela 3). Essa diferença talvez possa ser explicada pelo fato de que o controle de hábitos bucais deletérios implica basicamente em orientações aos pacientes e familiares, de modo que os CD se sentem mais aptos a intervir nesta situação, mesmo porque em Florianópolis tais orientações estão incorporadas ao Protocolo de Atenção à Saúde Bucal há bastante tempo ${ }^{17}$.

De todo o modo, os resultados revelam uma baixa frequência de profissionais capacitados para realização dos tratamentos ortodônticos, principalmente para os interceptativos. Tais considerações também foram ponderadas por Castro ${ }^{10}$, considerando os profissionais da $\mathrm{AB}$ experientes e capacitados para realização de diagnóstico, para desenvolver ações de orientação e algumas de prevenção, porém pouco capacitados para a confecção e a instalação de aparelhos ortodônticos, necessitando de apoio quanto ao 
Tabela 2. continuação

\begin{tabular}{|c|c|c|c|c|c|c|}
\hline & $\begin{array}{l}\text { Necess. } \\
\text { controle } \\
\text { hábitos } \\
\text { deletérios }\end{array}$ & $\begin{array}{c}\text { Aptidão } \\
\text { controle } \\
\text { hábitos } \\
\text { deletérios }\end{array}$ & $\begin{array}{c}\text { Necess. } \\
\text { trat. } \\
\text { M.C. }\end{array}$ & $\begin{array}{c}\text { Aptidão } \\
\text { trat. } \\
\text { M.C. }\end{array}$ & $\begin{array}{c}\text { Necess. } \\
\text { trat. } \\
\text { M.A. }\end{array}$ & $\begin{array}{c}\text { Aptidão } \\
\text { trat. } \\
\text { M.A. }\end{array}$ \\
\hline \multicolumn{7}{|c|}{ Tempo atuação profissional } \\
\hline correlação $\rho$ &,- 149 &,- 221 &, 108 &,- 125 &,- 063 &,- 219 \\
\hline significância $\mathbf{p}$ &, 243 &, 082 & ,398 &, 324 &, 620 &, 082 \\
\hline \multicolumn{7}{|l|}{ Tempo AB } \\
\hline correlação $\rho$ &,- 065 &,- 128 &, 129 &,- 139 &,- 051 &,- 199 \\
\hline significância $\mathbf{p}$ &, 615 & ,316 & ,310 &, 275 & ,692 &, 115 \\
\hline \multicolumn{7}{|c|}{ Necessidade manutenção espaço } \\
\hline correlação $\rho$ &, $363^{* *}$ &, 147 &, 031 &,- 033 & ,332 &, 115 \\
\hline significância p &, 004 &, 254 &, 811 & ,795 & ,008 & ,368 \\
\hline \multicolumn{7}{|c|}{ Aptidão manutenção espaço } \\
\hline correlação $\rho$ & ,099 &, 591 &,- 054 &, 552 & ,023 & ,662 \\
\hline significância $\mathbf{p}$ & ,444 & ,000 &, 675 & ,000 &, 857 &, 000 \\
\hline \multicolumn{7}{|c|}{ Necessidade rec. espaço } \\
\hline correlação $\rho$ &, $458^{* *}$ &, 182 & ,358 &, 047 & ,362 & ,098 \\
\hline significância $\mathbf{p}$ &, 000 & , 154 & ,004 & ,709 & ,003 & , 442 \\
\hline \multicolumn{7}{|l|}{ Aptidão rec. espaço } \\
\hline correlação $\rho$ &, 189 &, 540 & ,003 & ,758 & ,099 & ,745 \\
\hline significância p &, 138 & ,000 & ,981 & ,000 & ,434 & ,000 \\
\hline \multicolumn{7}{|c|}{ Necessidade controle hábitos deletérios } \\
\hline correlação $\rho$ & 1,000 &, 113 & ,489 &,- 001 &, 568 &, 150 \\
\hline significância $\mathbf{p}$ & . & ,380 & ,000 & ,994 & ,000 &, 241 \\
\hline \multicolumn{7}{|c|}{ Aptidão controle hábitos deletérios } \\
\hline correlação $\rho$ &, 113 & 1,000 &,- 008 &, 590 &, 070 & ,647 \\
\hline significância p & ,380 & . &, 950 & ,000 &, 584 & ,000 \\
\hline \multicolumn{7}{|c|}{ Necessidade trat. M.C. } \\
\hline correlação $\rho$ &, $489^{* *}$ &,- 008 & 1,000 &, 100 & ,497 &, 083 \\
\hline significância p &, 000 & ,950 & . & ,430 & ,000 &, 514 \\
\hline \multicolumn{7}{|l|}{ Aptidão trat. M.C. } \\
\hline correlação $\rho$ &,- 001 & ,590 & , 100 & 1,000 & ,042 &, 829 \\
\hline significância $\mathbf{p}$ & ,994 & ,000 & , 430 & . & ,743 & ,000 \\
\hline \multicolumn{7}{|c|}{ Necessidade trat. M.A. } \\
\hline correlação $\rho$ &, $568^{* *}$ &, 070 & ,497 &, 042 & 1,000 &, 225 \\
\hline significância $\mathbf{p}$ & ,000 &, 584 & ,000 & ,743 & . &, 073 \\
\hline \multicolumn{7}{|l|}{ Aptidão trat. M.A. } \\
\hline correlação $\rho$ &, 150 & ,647 &, 083 &, 829 &, 225 & 1,000 \\
\hline significância p &, 241 &, 000 &, 514 &, 000 &, 073 & . \\
\hline
\end{tabular}

M.C. $=$ mordida cruzada e M.A. $=$ mordida aberta. Em negrito, valores com significância estatística.

aporte técnico e à disponibilização de recursos para a definição de planos de tratamento específicos às oclusopatias.

Ainda acerca da aptidão dos profissionais, foi verificada uma correlação significativa e inversamente proporcional entre o tempo de atuação profissional e na $\mathrm{AB}$, com a aptidão para realizar tratamento de manutenção de espaço ( $\rho$ $=-0,366)$ e de recuperação de espaço $(\rho=$ $-0,250)$. Com os demais tratamentos, também houve essa correlação negativa, porém os valores não foram estatisticamente significativos (Tabela 2). Os resultados sugerem, desta forma, que quanto maior o tempo de atuação profissional e na $\mathrm{AB}$, menor a aptidão dos profissionais para realizar os tratamentos, assim como observado quanto à necessidade sentida pelos tratamentos ortodônticos preventivos e interceptativos. É possível que o foco na promoção da saúde e na prevenção de doenças das novas diretrizes curri- 
Tabela 3. Aptidão dos $\mathrm{CD}$ da rede de $\mathrm{AB}$ à saúde em realizar os tratamentos ortodônticos preventivos e interceptativos.

\begin{tabular}{|c|c|c|c|c|c|c|c|c|c|c|}
\hline & \multicolumn{2}{|c|}{ Man. espaço } & \multicolumn{2}{|c|}{ Rec. espaço } & \multicolumn{2}{|c|}{ C.H.B.D. } & \multicolumn{2}{|c|}{ Mord. cruzada } & \multicolumn{2}{|c|}{ Mord. aberta } \\
\hline & $\mathbf{F}$ & $\mathbf{P}$ & $\mathbf{F}$ & $\mathbf{P}$ & $\mathbf{F}$ & $\mathbf{P}$ & $\mathbf{F}$ & $\mathbf{P}$ & $\mathbf{F}$ & $\mathbf{P}$ \\
\hline Nada apto & 14 & 21,9 & 20 & 31,3 & 7 & 10,9 & 18 & 28,1 & 19 & 29,7 \\
\hline Pouco apto & 33 & 51,6 & 38 & 59,4 & 35 & 54,7 & 38 & 59,4 & 34 & 53,1 \\
\hline Muito apto & 12 & 18,8 & 3 & 4,7 & 14 & 21,9 & 4 & 6,3 & 6 & 9,4 \\
\hline Total. apto & 4 & 6,3 & 3 & 4,7 & 7 & 10,9 & 4 & 6,3 & 5 & 7,8 \\
\hline Total & 63 & 98,4 & 64 & 100,0 & 63 & 98,4 & 64 & 100,0 & 64 & 100,0 \\
\hline Perdas & 1 & 1,6 & - & - & 1 & 1,6 & - & - & - & - \\
\hline Total & 64 & 100,0 & 64 & 100,0 & 64 & 100,0 & 64 & 100,0 & 64 & 100,0 \\
\hline
\end{tabular}

$\mathrm{F}=$ frequência absoluta, $\mathrm{P}=$ percentual.

culares para os cursos de graduação da saúde ${ }^{18}$ seja responsável por esta diferença entre os profissionais mais jovens e os mais experientes, mas como ponderado anteriormente, haveria que se investigar esta e outras possíveis hipóteses explicativas a partir de abordagens metodológicas mais adequadas.

Cabe ainda mencionar que os cruzamentos entre a aptidão para realizar os diferentes tipos de tratamentos ortodônticos apresentaram correlações positivas com significância estatística (Tabela 2), permitindo concluir que quando os profissionais são mais ou menos aptos para realização de um determinado procedimento, também o são em relação aos demais. Já o cruzamento entre necessidade de realizar os diferentes tipos de tratamentos e a aptidão para realizá-los não mostrou nenhum tipo de correlação.

\section{Percepção quanto à diferença de relevância entre os tratamentos elencados e necessidade de realização de outros procedimentos ortodônticos na $\mathrm{AB}$}

Ao se questionar se haveria diferença na relevância entre os tratamentos indicados, $60,9 \%$ dos CD (39) responderam não haver e $39,1 \%$, haver diferença (25). Entre estes, houve uma concentração de respostas indicando o controle de hábitos bucais deletérios (36\%), a manutenção de espaço $(28 \%)$ e o tratamento de mordida cruzada $(20 \%)$ como sendo os mais relevantes.

Em relação aos hábitos bucais deletérios, um estudo transversal realizado com 2.139 crianças na faixa etária de 3 a 5 anos de Bauru (SP) mostrou a prevalência de má oclusão em $51,3 \%$ entre os meninos e $56,9 \%$ entre as meninas, sendo significantemente mais elevada no grupo etário de três anos. Entre os fatores ambientais estudados, o hábito de sucção de chupeta foi o mais importante na associação com má oclusão, seguido da sucção digital ${ }^{9}$.

Outro estudo realizado com crianças entre 3 e 5 anos em Araraquara (SP) aponta a influência de hábitos bucais na instalação de maloclusões na dentição decídua. Numa amostra de 329 crianças, foi encontrada a presença de hábitos de sucção em 194 delas, havendo 149 com alterações de oclusão ${ }^{19}$.

Essas pesquisas apontam o grande percentual de crianças com hábitos deletérios e a predominante influência destes no desenvolvimento das maloclusões, principalmente de mordida aberta e mordida cruzada $^{20}$, amparando a perspectiva dos CD de que controlar precocemente tais hábitos seria um procedimento de maior relevância em relação aos demais.

$\mathrm{Na}$ presente pesquisa, a maioria dos entrevistados, $81,3 \%$ (52), julgou desnecessária a realização de outros tipos de tratamentos ortodônticos preventivos e interceptativos na $\mathrm{AB}$ além dos mencionados no questionário. Os demais acharam necessários e sugeriram outros tratamentos, porém tais sugestões não foram contabilizadas por se enquadrarem no rol de procedimentos já elencados ou por não condizerem com procedimentos categorizados como preventivos e interceptativos pela literatura.

\section{Interesse quanto à realização de procedimentos ortodônticos preventivos e interceptativos nas UBS}

Quando perguntados se teriam interesse em realizar os tratamentos em discussão, $32,8 \%$ dos profissionais (21) responderam não ter, $64,1 \%$ 
deles (41) afirmaram ter interesse desde que fossem capacitados, e os demais $3,1 \%$ (2) indicaram ter interesse mesmo sem necessidade de receber capacitação, por já serem especialistas em Ortodontia.

Ao analisarmos as justificativas para o desinteresse de quase um terço dos entrevistados, observaram-se como motivos mais citados: sobrecarga de serviço - falta de tempo, excesso de demanda e falta de recursos humanos (10 justificativas); entendimento de que a ortodontia, mesmo a preventiva, não é responsabilidade da $\mathrm{AB}(5)$; falta de habilidade/capacitação profissional (4); profissionais em processo de aposentadoria (3); falta de recursos financeiros e de materiais (2); serviços já oferecidos ainda insatisfatórios ou insuficientes (2); e não gostar de realizar procedimentos ortodônticos (1).

Nas palavras dos profissionais, algumas das justificativas foram assim apresentadas:

Sei da importância deste tipo de tratamento, mas acredito que deva ser realizado por um profissional especializado. Outro problema é a sobrecarga da AB. Não adianta fazer leis e promessas e não ter recursos humanos para que de fato atenda a população de forma integral e resolutiva. (CD 6.2)

Penso que somente pessoas que não conhecem a realidade das UBS poderiam ter a ideia de fazer ortodontia preventiva. Ainda não demos conta dos outros procedimentos básicos, o CEO ainda não funciona de maneira completamente satisfatória, a demanda por serviços de urgência ainda é muito elevada. (CD 15)

Pela baixa prevalência, acho que a intervenção deve ser realizada totalmente na atenção secundária. Acho equivocada a politica do Ministério da Saúde quanto à abordagem da má oclusão na $A B$, além de aumentar o custo para o município com a descentralização deste serviço (equipamentos e materiais para confecção de aparelhos). Apesar do declínio da cárie e da doença periodontal, estas ainda são as mais prevalentes e, portanto, o foco da $A B$. (CD 19.2)

Já participo de varias atividades como PSE, visitas domiciliares, grupos de atenção. Não tenho tempo disponivel para isso. (CD 11)

Analisando-se especificamente a perspectiva de alguns $\mathrm{CD}$ de que os procedimentos discutidos deveriam ser realizados somente por profissionais especializados, observa-se uma discordância com a literatura que indica que a maior parte das ações ortodônticas preventivas e interceptativas pode ser feita pelo clínico geral, pois são de baixo custo e demandam menor dificuldade na sua execução ${ }^{20}$. A decisão do clínico geral de tratar ou encaminhar depende do conhecimento e da experiência individual, mas o esperado é que os problemas mais simples possam ser solucionados na $\mathrm{AB}$ e os problemas mais complexos, encaminhados para um especialista ${ }^{7,21}$.

Acerca das justificativas para o desinteresse dos profissionais atribuídas à sobrecarga $\mathrm{da} A \mathrm{~B}$ com as doenças bucais mais prevalentes, por conta do excesso de demanda, é possível se questionar que importância os mesmos atribuem ao princípio doutrinário da integralidade do SUS, ainda mais frente ao panorama anteriormente discutido de declínio da carie dentária na parcela mais jovem da população ${ }^{12}$. Certamente a falta de recursos humanos é um fator dificultador para a resolutividade da $\mathrm{AB}$, mas atribuir tal desinteresse à falta de tempo quando a carga horária diária de trabalho não se alteraria em função da maior ou menor integralidade da assistência realizada, não parece ser uma justificativa razoável. Talvez haja uma questão de motivação que pudesse ser trabalhada com esses profissionais, no sentido de despertar seu interesse para os procedimentos ortodônticos possíveis de serem realizados na $A B$ e para a integralidade do cuidado. A disponibilização de uma estrutura física adequada, a definição de protocolos de tratamento, além de capacitação profissional e uma adequada gestão do serviço ${ }^{7,10}$ poderiam ser fatores a contribuir nesse sentido.

\section{Aspectos que poderiam dificultar a introdução de procedimentos ortodônticos preventivos e interceptativos nas UBS}

Com a finalidade de se prever possíveis barreiras para a realização da ortodontia preventiva e interceptativa na rede, formulou-se o seguinte questionamento: "Supondo que a Secretaria Municipal de Saúde fornecesse todas as condições necessárias à ampliação dos procedimentos ortodônticos preventivos e à implantação dos procedimentos ortodônticos interceptativos nas UBS, como disponibilização de serviços laboratoriais e de documentação ortodôntica, pessoal auxiliar, materiais de consumo, capacitações profissionais e oferta de tratamentos ortodônticos corretivos no CEO, que outros aspectos poderiam dificultar a viabilização da realização desses procedimentos"?

Os fatores indicados como possíveis dificultadores foram: excesso de demanda (18); falta de recursos humanos (13); precariedade da $A B$ ou falta de resolutividade (11); falta de adesão e/ 
ou manutenção do tratamento ortodôntico (8); falta de capacitação e/ou habilidade dos CD e falta de pessoal auxiliar (7); falta de interesse profissional por parte dos CD (4); procura por tratamento ortodôntico "apenas" por estética (3); aspectos logísticos relacionados a problemas com os serviços laboratoriais (tempo/fluxo), deslocamento de pacientes e da documentação ortodôntica (3); baixa remuneração profissional (2); falta de recursos financeiros (2); serviços de referência e contrarreferência ineficazes (2); falta de espaço e estrutura física (2); necessidade de materiais de qualidade adequada (2); dificuldade em determinar critérios de seleção (2); entre outros.

Nas palavras dos profissionais:

A demanda. A ortodontia, em virtude de condicionantes relativamente complexos, tornou-se uma moda. São questionáveis e discutíveis quais são os critérios que indicam ou não o tratamento ortodôntico, sendo que no mais das vezes são critérios bastante subjetivos (estéticos).... (CD 44)

Na própria $A B$, a falta de recursos humanos no setor de regulação (onde se encaminha o paciente para a atenção especializada), já dificulta o encaminhamento a qualquer serviço especializado. (CD 23.2)

A desproporção entre equipes de saúde bucal e equipes de saúde da família, que em Florianópolis faz com que muitas equipes de saúde bucal trabalhem com uma população muito grande [...] faz com que a demanda seja cada vez maior e o dentista não consegue dar acesso a toda população que necessita de intervenções clínicas e nem conciliar com suas outras atribuições da AB. (CD 14)

A oferta deste tipo de tratamento e o volume da necessidade verificada na população são dispares. Como certamente haverá síndrome do cobertor curto seria fundamental a delimitação de população prioritária ou de risco [...]. (CD 24)

Em ortodontia é muito importante que seja feito o acompanhamento mensal do paciente, pelo mesmo profissional, sem trocar. Então teríamos quer ter recursos humanos de ortodontistas o suficiente para garantir o tratamento ideal, pois o tratamento é uma sequência de etapas que variam de técnica para técnica e conduta profissional diferente. Os materiais devem ser de qualidade, pois a questão força é muito importante, no intuito de evitar problemas periodontais e reabsorções radiculares indesejadas. Ortodontia é muito sério, tem que ter sequência e conscientização do paciente também (50\% é de responsabilidade do paciente de comparecer a consulta mensal). Temos que ter um bom controle de cárie nestes pacientes, pois aparelhos retêm mais alimentos, então a seleção dos pacientes aptos ao tratamento é muito importante [...] Ortodontia é bem complexo, pois tratar é fácil, difícil é garantir que não haja recidiva com contenções eficientes e cooperação do paciente. (CD 35)

Como se pode ver, algumas barreiras apontadas pelos CD já estavam contempladas no enunciado da questão, uma vez que se sabe de antemão de sua importância para a efetivação da oferta deste tipo de serviço. A reafirmação de tais questões nas respostas, no entanto, parece indicar uma descrença na real possibilidade da reunião de todas as condições elencadas. Da mesma forma, ao reapresentarem determinados argumentos, alguns profissionais parecem ter reiterado seu desinteresse em realizar os procedimentos ortodônticos preventivos e interceptativos.

Problemas com materiais e com a realização de exames complementares são possíveis de serem evitados ou ao menos minimizados, a partir de uma clara definição dos papéis de cada nível e setor da rede de atenção em saúde bucal, com estudos de viabilidade econômica, com gestão adequada e com a definição e o emprego de protocolos de atendimento em cada município ${ }^{10}$.

Outros fatores dificultadores mencionados poderiam ser resolvidos a partir das próprias condições explicitadas na formulação da pergunta, como por exemplo, a dificuldade em se determinar critérios para a seleção de pacientes, pois a partir das capacitações profissionais, critérios técnicos poderiam ajudar nessa tarefa. A propósito, e para além dos índices, Hebling et al. ${ }^{7}$ propõem critérios de inclusão na definição de prioridades de atendimento, tais como assiduidade, condições de saúde bucal, qualidade da higienização, condições econômicas etc., enquanto Peres et al. ${ }^{16}$ alertam para a importância de se considerar a autopercepção dos sujeitos, uma vez que existem graus de problemas oclusais definidos tecnicamente que são aceitáveis pela população e que devem ser considerados na decisão de indicação de tratamento ortodôntico, principalmente nos serviços públicos.

Retomando os fatores dificultadores apontados, haveria outros ainda que demandariam discussão e deliberação entre os CD da rede e nas instâncias de controle social do SUS, mas nenhuma das possíveis barreiras elencadas parece ser realmente instransponível se o estabelecimento de critérios para acesso ao serviço forem consensuados, se protocolos de assistência forem definidos e adotados, e se a gestão dos recursos físicos, humanos e financeiros for bem conduzida ${ }^{7}$. 
Aspectos positivos sobre a possível ampliação da oferta de procedimentos ortodônticos preventivos e disponibilização de procedimentos ortodônticos interceptativos nas UBS

Quando questionados sobre as vantagens de se ampliar e de se implementar procedimentos de ortodontia na $\mathrm{AB}$ os $\mathrm{CD}$ mencionaram: prevenção de patologias bucais (23); integralidade da atenção à saúde (16); melhora da saúde e/ou qualidade de vida (10); universalidade e/ou ampliação do acesso (10); resolutividade da assistência (8); melhora da "saúde bucal" (7); melhora da estética (6); efetivação do direito à saúde (4); ampliação dos postos de trabalho para CD (3); satisfação profissional (2); ampliação da atenção secundária e possibilidade de referenciar para a ortodontia como serviço de média complexidade (2); valorização da Odontologia (1); e ausência de aspectos positivos para a coletividade (1).

Nas palavras dos CD:

Estaríamos evitando tratamentos radicais na fase adulta com exodontias ou cirurgias se tratássemos as crianças na fase de crescimento adequado. Nos adultos, ofereceríamos condições estéticas e funcionais ideais para uma boa saúde bucal, pois dentes com apinhamentos ou com diastemas, por exemplo, são difíceis de higienizar, aumentando riscos de cáries. Isso tudo está no que chamamos no SUS de atendimento integral do paciente. É muito importante que o SUS ofereça este serviço, assim como próteses, pois com certeza isto interfere nas condições sociais das pessoas, pois o sorriso é o cartão de visita. Ter um sorriso saudável implica em acesso a empregos, melhor remuneração e melhor convívio social e, também, interfere nos relacionamentos interpessoais. (CD 35)

Consolidação dos princípios do SUS; satisfação profissional; valorização da categoria odontológica com ampliação do mercado de trabalho do SUS; qualidade de vida para os usuários. (9.1)

Seria ótimo se tivéssemos a ortodontia na rede de atenção, pois o serviço só iria complementar o que é oferecido pelo SUS, dando mais resultados ao serviço. (CD 23.2)

Só vejo aspectos positivos para os pacientes e para nós profissionais: menor tempo para o tratamento corretivo, melhora na incidência de cárie por diminuição do apinhamento etc. (CD 13.3)

Cumprimento dos direitos civis garantidos em lei pela constituição cidadã. (CD 23.1)
Considerando que buscamos melhorar a saúde da população, acho fantástico! (CD 20)

Positivos? Somente para os poucos que forem contemplados e que levarem o tratamento até o final. Pode parecer pessimismo, mas depois de $x$ anos trabalhando no serviço público, estadual $e$ municipal, penso que tudo que é dado sem a contrapartida de responsabilidade do recebedor Não funciona, basta se verificar a quantidade de consultas especializadas que são perdidas (tanto na área de odontologia, como médica), os tratamentos que são interrompidos (pelas mais diversas razões), a falta de adesão a tratamentos nas mais diversas áreas. É bom dizer que mantive consultório particular por 15 anos, portanto tenho experiências nas duas iniciativas. (CD 15)

Vários dos aspetos positivos apontados pelos CD estão de acordo com a literatura e vão ao encontro da Política Nacional de Saúde Bucal, como a prevenção de patologias; a integralidade da atenção à saúde; a melhora da saúde e/ou da qualidade de vida; a universalidade e/ou a ampliação do acesso; a resolutividade da assistência, entre outros.

A ampliação e a qualificação da $\mathrm{AB}$ estão contempladas também nas diretrizes da Política Nacional de Saúde Bucal visando à ampliação da oferta de serviços e buscando a integralidade da atenção, além da equidade e a universalização do acesso às ações ${ }^{22}$. Nessa direção, a rede de $\mathrm{AB}$ é uma ótima alternativa para disponibilizar uma quantidade maior de serviços e ampliar o atendimento às necessidades da população, incluindo nas Unidades Básicas de Saúde serviços mais especializados, o que nos leva a vislumbrar um país com possibilidades de suprir as necessidades de saúde da população ${ }^{23}$.

Vale ainda lembrar que o conceito ampliado de saúde da Constituição Federal a considera dentro de um modelo de atenção integral que prevê a incorporação progressiva de ações de promoção e de proteção à saúde, em conjunto com as ações propriamente ditas de recuperação ${ }^{23}$. Neste sentido, a ampliação da ortodontia preventiva e a implementação da ortodontia interceptativa na $\mathrm{AB}$ à saúde é altamente desejável, epidemiologicamente justificável e, para além de todos os benefícios mencionados, seria mais uma das formas do SUS fazer valer o direito de cidadania assegurado em seu arcabouço legal. 


\section{Considerações finais}

De acordo com os profissionais entrevistados é desejável que a população disponha de tratamentos ortodônticos preventivos e interceptativos no SUS, bem como dos benefícios que essa ampliação na cobertura da assistência à saúde lhe traria. Mas, apesar da maioria se posicionar favoravelmente e demonstrar interesse na realização dos procedimentos discutidos na $\mathrm{AB}$, muitos se consideram pouco ou nada aptos para a sua realização. Assim, ainda que se trate de procedimentos básicos de ortodontia, seriam necessários cursos de capacitação e/ou atualização para os profissionais da rede estudada.

Essa conclusão remete a um questionamento imediato sobre a qualidade da formação acadêmica em nossos cursos de Graduação em Odontologia, que se revela no despreparo dos profissionais para diagnosticar, tratar e encaminhar pacientes com necessidades ortodônticas ${ }^{7,24}$. Ao mesmo tempo, aponta para a necessidade de se continuar com os esforços de integração ensinoserviço já iniciados e de ampliá-los no sentido de um maior apoio institucional e da participação e valorização do quadro docente como um todo, para que, ao final, o formando se sinta mais familiarizado e comprometido com o SUS, capacitado a atuar na transformação do seu modelo de atenção, de gestão e de controle social ${ }^{25}$.

Além de uma adequada formação aos novos profissionais e da educação permanente aos que se encontram inseridos na rede, um protocolo de prevenção e interceptação às oclusopatias para a inclusão em programas de saúde bucal, no âmbito da $A B$ se faz fundamental. Seria, portanto, interessante que cada município que considere viável a realização de ações ortodônticas preventivas e interceptativas nas Unidades Básicas de Saúde, com base na literatura e juntamente com os profissionais da rede e de seus usuários, estruturassem seus próprios protocolos de atendimento ortodôntico, adequados às principais demandas de cada município.

Indo além, a oferta desses serviços na $\mathrm{AB}$ demandaria também a estruturação da sua oferta na atenção secundária, de modo a possibilitar a integralidade da atenção à saúde e a resolutividade da assistência. Sabe-se do tamanho do desafio, mas o momento parece ser bastante oportuno, principalmente pela recente publicação da Portaria $\mathrm{n}^{\circ}$ 718/SAS, de 20 de dezembro de 2010, que incorporou na relação de serviços que podem ser ofertados pelo SUS, os procedimentos ortodônticos, antes limitados a pacientes com anomalias cranianas e bucomaxilofaciais ${ }^{11}$. Cabe, portanto, a cada município, estruturar-se para a ampliação e qualificação de seus serviços.

Diante de todo o exposto, reafirma-se a inclusão dos tratamentos ortodônticos preventivos e interceptativos nas UBS como desejável, apropriada e possível de ser realizada, acreditando-se na tendência apontada de que o tratamento das oclusopatias, principalmente as de soluções ortodônticas mais simples, deva estar presente rotineiramente na assistência à saúde do SUS7 ${ }^{7}$

\section{Colaboradores}

SC Guzzo idealizou a pesquisa, coletou, analisou os dados e redigiu o trabalho. M Finkler idealizou e orientou a pesquisa, redigiu e revisou o trabalho. C Reibnitz Júnior e MT Reibnitz analisaram criticamente o trabalho e revisaram o artigo. 


\section{Referências}

1. Brasil. Ministério da Saúde (MS). Secretaria de Atenção à Saúde. Departamento de Atenção Básica. Guia de recomendações para o uso de fluoretos no Brasil. Brasília: MS; 2009. (Normas e Manuais Técnicos. Série A)

2. World Health Organization (WHO). Health Through Oral Health: Guidelines for Planning and Monitoring for Oral Health Care. London: Quintessence; 1989.

3. Simões WA. Prevenção das oclusopatias. Ortodontia; 1978; 11:117-125.

4. Schinestsck P, Schinestsck AR. A importância do tratamento precoce da má-oclusão dentária para o equilíbrio orgânico e postural. J. Bras. Ortodontia Ortop. Maxilar 1998; 3(13):15-30.

5. Dracker HL. Handicapping labio-lingual deviations: a propose index for public health purposes. Am. J. Orthodontics 1960; 46(4):295-305.

6. Salzman JA. Principles of orthodontics. Philadelphia: J. B. Lippincott; 1943.

7. Hebling SRF, Pereira AC, Hebling E, Meneghim MC. Considerações para elaboração de protocolo de assistência ortodôntica em saúde coletiva. Cien Saude Colet 2007; 2(4):1067-1078.

8. Lopes-Monteiro S, Nojima MCC, Nojima LI. Ortodontia Preventiva x Ortodontia Interceptativa: Indicações e Limitações. J. Bras. Ortodon. Ortop. Facial 2003; 7(47):390-397.

9. Tomita NE, Bijella VT, Franco LJ. Relação entre hábitos bucais e má oclusão em pré-escolares. Rev Saude Publica 2000; 34(3):299-303.

10. Castro RG. Diretrizes para a atenção às oclusopatias no sistema único de saúde [tese]. Florianópolis: Universidade Federal de Santa Catarina; 2010.

11. Brasil. Ministério da Saúde (MS). Secretaria de Atenção à Saúde. Departamento de Atenção Básica. Coordenação Geral de Saúde Bucal. Portaria SAS 718: nota técnica. Brasília: MS; 2010.

12. Brasil. Ministério da Saúde (MS). Secretaria de Atenção à Saúde/Secretaria de Vigilância em Saúde. Departamento de Atenção Básica. Coordenação Geral de Saúde Bucal. Projeto SB Brasil 2010 - Pesquisa Nacional de Saúde Bucal: Resultados Principais. Brasília: Ministério da Saúde; [página na Internet] 2011. [acessado 2012 nov 26]. Disponível em: http://dab. saude.gov.br/cnsb/sbbrasil/arquivos/projeto_sb 2010_relatorio_final.pdf

13. Frasão P, Narvai PC, Latorre MRDO, Castellanos RA. Prevalência de oclusopatia na dentição decídua e permanente de crianças na cidade de São Paulo, Brasil, 1996. Cad Saude Publica 2002; 18(5): 1197-1205.

14. Lopes LS, Cangussu MCT. Prevalência e severidade das alterações oclusais em escolares de 12 a 15 anos de Salvador - Bahia, Brasil, 2004. Rev. Cienc. Med. Biol. 2005; 4(2):105-112.

15. Marques LS, Barbosa CC, Ramos-Jorge ML, Pordeus IA, Paiva SM. Prevalência da maloclusão e necessidade de tratamento ortodôntico em escolares de 10 a 14 anos de idade em Belo Horizonte, Minas Gerais, Brasil: enfoque psicossocial. Cad Saude Publica 2005; 21(4):1099-1106.
16. Peres KG, Traebert ESA, Marcenes W. Diferenças entre autopercepção e critérios normativos na identificação de oclusopatias. Rev Saude Publica 2002; 36(2):230-236

17. Florianópolis. Prefeitura Municipal de Florianópolis. Secretaria de Saúde e Desenvolvimento Social. Programa capital criança: gestão 1997 a 2000. Florianópolis: Prefeitura Municipal de Florianópolis; 1997.

18. Brasil. Ministério da Educação (MEC). Conselho Nacional de Educação. Câmara de Educação Superior. Resolução CNE-CES no 3, de 3 de março de 2002. Institui as Diretrizes Curriculares Nacionais do Curso de Graduação em Odontologia. Diário Oficial da União 2002; 4 mar.

19. Zuanon ACC, Oliveira MF, Giro EMA, Maia JP. Relação entre hábito e maloclusão na dentadura decídua. J. Bras. Odontopediatr. Odontol. Bebe 2000; 3(12):104-108.

20. Maciel SM, Kornis GEM. A ortodontia nas políticas públicas de saúde bucal: um exemplo de equidade na Universidade Federal de Juiz de Fora. Physis 2006; 16(1):59-81.

21. Proffit WR. Plano do tratamento ortodôntico: da lista de problemas ao plano específico. In: Proffit WR, organizador. Ortodontia Contemporânea. Rio de Janeiro: Elsevier; 2007. p. 217-248.

22. Brasil. Ministério da Saúde (MS). Secretaria de Atenção à Saúde. Departamento de Atenção Básica. Caderno de Atenção Básica no 17: Saúde Bucal. Brasília: MS; 2008.

23. Brasil. Ministério da Saúde (MS). Secretaria de Atenção à Saúde. Departamento de Atenção Básica. Coordenação Nacional de Saúde Bucal. Diretrizes da Política Nacional de Saúde Bucal. Brasília: MS; 2004.

24. Maciel SM. A presença da ortodontia no SUS: a experiência dos CEOs e de outros serviços públicos de saúde bucal [tese]. Rio de Janeiro: Universidade do Estado do Rio de Janeiro; 2008.

25. Finkler M, Caetano JC, Ramos FRS. Integração ensino-serviço no processo de mudança na formação profissional em Odontologia. Interface (Botucatu) 2011; 15(39):1053-1067.

Artigo apresentado em 30/11/2012

Aprovado em 16/03/2013

Versão final apresentada em 26/03/2013 